\title{
Myanmarforschung 2015: Interdisziplinäre Tagung zur Vernetzung der deutschsprachigen Myanmarforschenden
}

\author{
Winterberger, Georg
}

\begin{abstract}
Myanmar is facing a phase of change. The opening of the country in 2011 has consequences on the political, economic and social level. This phase is very complex and not yet thoroughly explored and understood, therefore, we find a growing number of scientists who do or plan research in Myanmar. The conference "Myanmarforschung 2015 - Interdisziplinäre Tagung zur Vernetzung der deutschsprachigen Myanmarforschenden" (Myanmar research 2015 - interdisciplinary conference for networking of German speaking Myanmar researchers), which was held from July 23th to 25th 2015 at the University of Zurich, had the aim to give a platform to these scientists for sharing their knowledge. This paper gives an overview and background information about this conference and it reports about the presentations, which were held in Zurich.
\end{abstract}

DOI: https://doi.org/10.1515/asia-2016-0005

Posted at the Zurich Open Repository and Archive, University of Zurich ZORA URL: https://doi.org/10.5167/uzh-125478

Journal Article

Published Version

Originally published at:

Winterberger, Georg (2016). Myanmarforschung 2015: Interdisziplinäre Tagung zur Vernetzung der deutschsprachigen Myanmarforschenden. Asiatische Studien / Études Asiatiques, 70(1):247-258.

DOI: https://doi.org/10.1515/asia-2016-0005 
Georg Winterberger*

\title{
Myanmarforschung 2015: Interdisziplinäre Tagung zur Vernetzung der deutschsprachigen Myanmarforschenden
}

DOI 10.1515/asia-2016-0005

\begin{abstract}
Myanmar is facing a phase of change. The opening of the country in 2011 has consequences on the political, economic and social level. This phase is very complex and not yet thoroughly explored and understood, therefore, there is a growing number of scientists who do or plan research in Myanmar. The conference "Myanmarforschung 2015 - Interdisziplinäre Tagung zur Vernetzung der deutschsprachigen Myanmarforschenden” (Myanmar research 2015 - interdisciplinary conference for networking of German speaking Myanmar researchers), which was held from July $23^{\text {th }}$ to $25^{\text {th }} 2015$ at the University of Zurich, had the aim to give a platform to these scientists for sharing their knowledge. This paper gives an overview and background information about this conference and it reports about the presentations, which were held in Zurich.
\end{abstract}

Keywords: conference report, Myanmar, Burma, research, University of Zurich

Der südostasiatische Staat Myanmar/Burma ${ }^{1}$ befindet sich in einer Phase der Veränderung. ${ }^{2}$ Diese Öffnung seit 2011 hat weitreichende politische, wirtschaftliche und soziale Folgen und zieht somit das Interesse der WissenschaftsCommunity auf sich. Forschende zieht es gerade jetzt nach Myanmar, weil erst durch die Öffnung qualitativ tiefgehende Forschung möglich wurde. ${ }^{3}$ Nicht zuletzt lässt sich gerade jetzt der Wandel von einer recht isolierten in eine

1 Viele Länder haben nach der Kolonialzeit ihre vorherige Staatsbezeichnung wieder angenommen, in diesem Fall wurde Burma wieder zu Myanmar. Kaum eine Namensänderung dürfte jedoch zu so vielen politischen Missverständnissen geführt haben wie die Bezeichnung Myanmar (Steinberg 2013: xx-xxi). In diesem Beitrag wird die heutige offizielle Staatsbezeichnung Myanmar verwendet.

2 Myint-U 2014: 11.

3 Hlaing 2008; Selth 2010.

*Corresponding author: Georg Winterberger, Fach Ethnologie, Institut für Sozialanthropologie und Empirische Kulturwissenschaft, Universität Zürich, Andreasstrasse 15, 8050 Zürich, Schweiz, E-mail: georg.winterberger@uzh.ch. 
vernetzte, nun globalen Einflüssen und Strömen ausgesetzten Gesellschaft besonders gut beobachten.

Die Wissenschaftlerinnen und Wissenschaftler, die zu Myanmar forschen, verteilen sich über verschiedene Forschungsinstitutionen, und sie gehören verschiedenen Disziplinen an. Um diese Forschenden aus dem deutschsprachigen Raum zusammenzubringen, fand an der Universität Zürich vom 23. bis 25. Juli 2015 die Tagung „Myanmarforschung 2015 - Interdisziplinäre Tagung zur Vernetzung der deutschsprachigen Myanmarforschenden“ statt. Dieser Beitrag bietet einen Überblick über die Tagung und die Forschungsthemen.

Die Organisation der Tagung wurde getragen durch die Myanmarforschenden der Universität Zürich, ein loser Verbund, der 2014 vom Autor aufgrund der wachsenden Zahl der Forschungen zu Myanmar an der Universität Zürich, sowie aufgrund der Unterzeichnung eines Memorandum of Understanding zwischen der Universität Zürich und der Mandalay University in Myanmar im November 2014 ins Leben gerufen wurde. ${ }^{4}$ Auf dieser Website werden verschiedene Forschungen wie das Projekt ,The Greater Burma Zone - a transitional zone of languages and peoples“ am Institut für Vergleichende Sprachwissenschaft, an dem Mathias Jenny, Patrick McCormick, André Müller und Rachel Weymuth mit eigenen Subprojekten beteiligt sind, oder das Projekt „Strategien zur Existenzsicherung in Myanmar“ am ISEK - Ethnologie vom Autor, Georg Winterberger, bekannt gemacht. Des Weiteren finden Projekte wie „World Development“ am Soziologischen Institut und Forschungsmöglichkeiten in Zusammenarbeit mit dem Rietberg Museum in Zürich Erwähnung, das eine noch unbearbeitete Sammlung vom Sammler Toni Gerber (1932-2010) mit etwa 1500 Artefakten darunter viele aus Myanmar - erhalten hat.

„Myanmarforschung 2015“ konnte als Tagung nur dank der finanziellen Unterstützung des Graduate Campus der Universität Zürich, der Vereinigung akademischer Mittelbau der Universität Zürich (VAUZ) und der Hochschulstiftung der Universität Zürich durchgeführt werden. Gedankt sei auch für die Unterstützung des Faches Ethnologie am Institut für Sozialanthropologie und Empirische Kulturwissenschaft der Universität Zürich (ISEK), im Speziellen darin dem Lehrstuhl von Annuska Derks, und dem Völkerkundemuseum der Universität Zürich.

Die Organisation der Tagung geschah federführend durch den Autor und innerhalb der Myanmarforschenden der Universität Zürich. Unterstützung - und sozusagen historische Kontinuität - erhielt die Organisation der Tagung vom Lehrstuhl von Rüdiger Korff an der Universität Passau, vor allem durch Sascha Helbardt und Dagmar Hellmann-Rajanayagam. Historische Kontinuität deshalb,

4 Siehe www.myanmar.uzh.ch (02.09.2015). 
weil an der Universität Passau im Januar 2012 das Wissenschaftsforum Myanmar (WiMya) gegründet wurde, als „[...] ein Personennetzwerk mit dem Ziel, Wissenschaftler mit Myanmar-Bezug im deutschsprachigen Raum zu vernetzen und Synergien im Bereich Forschung und Wissenschaftskooperation $\mathrm{zu}$ schaffen, Zusammenarbeit mit Kollegen in Myanmar zu unterstützen, sowie Kontakte zu in Myanmar aktiven Organisationen herzustellen“. ${ }^{5}$ Das WiMya organisierte vom 5. bis 6. Januar 2012 den Workshop „Perspektiven der Myanmar-Forschung im deutschsprachigen Raum. Veranstaltung zur Vernetzung deutscher und österreichischer Wissenschaftler in der Myanmar-Forschung“ und vom 13. bis 14. Dezember 2013 die „WiMya Lecture Series Myanmar“. Anknüpfend daran fand am 27. Juni 2014 in Halle die von Felix Girke und Judith Beyer organisierte Tagung „Myanmarforschung 2014 - Projekte, Pläne, Partnerschaften“ am Zentrum für Interdisziplinäre Regionalstudien (ZIRS) an der Martin-LutherUniversität Halle-Wittenberg statt. ${ }^{6}$

\section{Tagungsbericht}

Der folgende Bericht führt gemäss Tagungsprogramm chronologisch durch die Beiträge der „Myanmarforschung 2015 - Interdisziplinäre Tagung zur Vernetzung der deutschsprachigen Myanmarforschenden“ an der Universität Zürich.

Mareile Flitsch, Direktorin des Völkerkundemuseums der Universität Zürich und Lehrstuhlinhaberin am ISEK - Ethnologie, betont in ihrer Begrüssungsrede, die sie in Vertretung von Annuska Derks hält, dass vor wenigen Jahren in der Ethnologie noch händeringend nach sprachkompetenten Myanmarforschenden gesucht wurde. Dieser Zustand habe sich - wie man auch am regen Interesse der Tagung sehe - verbessert. Aktuell sei die wissenschaftliche Beschäftigung mit Myanmar insbesondere auch deshalb wichtig, weil sich um Myanmar - bedingt durch die jahrelange Abschottung - zahllose Gerüchte, wenn nicht sogar Mythen, rankten. Es brauche deshalb dringend mehr aufschlussreiche und qualitätsvolle Forschungen und Publikationen zu Myanmar, dies natürlich auch aus der Ethnologie. Mareile Flitsch regt aufgrund mehrerer Anfragen aus der Öffentlichkeit an das Völkerkundemuseum ausserdem an, seitens der Myanmarforschenden einmal Stellung zur Lage der Rohingya zu nehmen. Sie plant, in den kommenden Jahren Wissenschaftlerinnen und Wissenschaftler,

5 Universität Passau 2015.

6 Die Programme und Berichte dieser Tagungen sind zu finden unter: http://www.myanmar. uzh.ch/events/tagung/archive.html (02.09.2015). 
die sich mit Myanmar befassen, darunter auch solche, die sich mit dem Thema Rohingya befassen, im Rahmen des öffentlichen Programms des Völkerkundemuseums der Universität Zürich einzuladen, um die Öffentlichkeit über Myanmar zu informieren.

Im Anschluss an die offizielle Begrüssung durch Yasmine Inauen im Namen des Rektors und der Universitätsleitung eröffnet Uta Gärtner von der HumboldtUniversität zu Berlin die Tagung mit einem historischen Überblick über die Myanmarforschungen im deutschsprachigen Raum. Sie betont jedoch, dass neben der Forschung die Lehre essentiell sei, denn ohne gut ausgebildete und vor allem sprachkompetente Nachwuchsforschende sei gute Forschung nicht möglich.

Den inhaltlichen Teil der Tagung leitet Tilman Frasch von der Manchester Metropolitan University mit seinem Referat „Pali in Bagan: Die lingua franca der buddhistischen Ökumene“ ein. Es handelt von der Stadt Bagan, die über 250 Jahre lang die Hauptstadt des Gebietes war, das sich in etwa mit dem heutigen Myanmar deckt. Aufbauend auf seine Arbeiten zu den Beziehungen zwischen Bagan, Sri Lanka und Nordindien zeigte er auf, dass das buddhistische Netzwerk auch Kambodscha mit einschloss. Mehr noch, es kann davon ausgegangen werden, dass die Stadt Bagan ein kosmopolitisches Zentrum des TheravadaBuddhismus gewesen sein muss, in dem sich Mönche und Gläubige aus ganz Asien trafen. Und diese Begegnungen von Menschen aus Myanmar, Sri Lanka, Kambodscha oder Nord- und Südindien setzen eine gemeinsame Verkehrssprache voraus. Diese muss Pali gewesen sein, die kanonische Sprache des Theravada-Buddhismus. Die Belege dafür sind jedoch nur dünn gesät und beschränkten sich bisher auf die wenigen literarischen Werke, die uns erhalten geblieben sind. Dazu treten inzwischen aber vermehrt auch in Pali abgefasste Stiftungs- bzw. Widmungsinschriften wie jene beiden, die vor etwa 15 Jahren neu gefunden wurden. Die in den Inschriften genannten auswärtigen Mönche und Laien erlauben es, die Rolle des Pali als lingua franca deutlich herauszuarbeiten.

Felix Hessler von der Leibniz Universität Hannover stellt in seinem Referat sein Promotionsprojekt zum Thema „Klosterschulen in Myanmar - Eine Analyse aktueller Transformationsprozesse“ vor: Die Klöster seien die einzigen Bildungsinstitutionen im vorkolonialen Birma gewesen. In der sogenannten „Economy of Merit“, 7 dem idealtypischen Beziehungssystem zwischen König, Mönchen und Laien, sei dem Sangha, also der Mönchsgemeinschaft, damals nicht nur die Aufgabe zugefallen, den Laien den Erwerb religiösen Verdienstes zu ermöglichen, sondern auch der Bevölkerung Bildung $\mathrm{zu}$ vermitteln. Obwohl die

7 Tambiah 1975; Schober 2011. 
Kolonialregierung ein säkulares Bildungssystem eingeführt habe, hätten die Klosterschulen fortbestanden. In jüngerer Zeit nehmen Klosterschulen vermehrt neue Rollen ein und erweitern ihren Einflussbereich durch Zusammenarbeit mit internationalen Organisationen und durch den Aufbau nationaler Netzwerke. Es gebe viele Klosterschulen, die heute das staatliche Curriculum unterrichten und in denen die Schülerinnen und Schüler kaum im Buddhismus unterwiesen werden. Diese fungierten damit als säkulare Bildungsinstitutionen, würden aber von Mönchen geleitet. Diese neueren Entwicklungen deuteten auf eine gesellschaftliche Neuverortung des Sangha - oder zumindest eines Teiles davon - und auf veränderte Beziehungsstrukturen innerhalb des „Economy of Merit“ Systems (Staat, Sangha, Laien).

Im Fokus des Referats „Rituale als Atmosphären. Musik in Besessenheitspraktiken Süd Myanmars“ von Friedlind Riedel von der Georg-August Universität Göttingen stehen die Nat Pwes, Verehrungsrituale oder Festveranstaltungen zu Ehren von Geistern, wie sie im Süden Myanmars verbreitet sind. Diese beginnen und enden jeweils mit durchdringender und ohrenbetäubender Musik, meist elektronisch verstärkt. Auch während der Rituale bzw. Feste wird die Musik zur Verstärkung und Untermalung der Handlungen benutzt. Riedel legt in ihrer Promotionsforschung einen besonderen Fokus auf die musikalischen Elemente der Rituale und auf die Effekte der Musik. Gleichzeitig betont sie, dass der alleinige Fokus auf die Musik das entscheidende Moment des Atmosphärischen vernachlässige. Unter ,Atmosphäre‘ versteht Riedel die Stimmung der Ritualsituation und das gesamte das Ritual umspannende Geschehen, das nicht an einzelnen Dingen oder Bedeutungen festzumachen ist.

André Müller und Rachel Weymuth promovieren im Rahmen des SNF-Projekts „The Greater Burma Zone - a transitional zone of languages and peoples“ an der Universtität Zürich. Das Projekt befasst sich mit der Linguistik und Geschichte Myanmars und der angrenzenden Gebiete, insbesondere der Interaktion zwischen Sprechern verschiedener Sprachen. In ihrem Referat „Sprache und Gesellschaft in Myanmar: Pronominalsysteme“ stellen Müller und Weymuth Gemeinsamkeiten im System der Personalpronomen einiger Sprachen der Region auf. Sie unterscheiden zwei unterschiedliche Typen: So hänge im hierarchischen Typ der Begriff für „ich“ etwa von der Person ab, die der Sprecher anspricht (gegenüber einem Freund, einem Mönch oder dem König wird jeweils ein anderes Wort für „ich“ benutzt). Im zweiten Typ werde dagegen immer dasselbe Wort benutzt. Eine Übersichtskarte, die die Verteilung der Sprachen beider Typen darstellt, zeigt, dass die Ebenen und Täler von Sprechern des hierarchischen Typs bewohnt sind, die Berge von Sprechern des ,grammatikalischen“ Typs. Als mögliche Erklärung wird genannt, dass in den Bergen kaum grössere Siedlungen zu finden sind und Sprecher sich üblicherweise persönlich 
kennen, wodurch sich eine stark abgestufte hierarchische Gliederung der Sprache erübrige. Wieso die Mon, die früher ein viel grösseres Gebiet beherrschten, kein komplexes hierarchisches System, sondern eine Art Mischsystem aus beiden besitzen, ist unter anderem Gegenstand des Forschungsprojektes.

Das Referat von Delphine Desoutter von der Sorbonne Nouvelle (Paris) Universität handelt „Von Terrakottatafeln bis zu bronzenen Stempeln: Einführung in eine buddhistische Praxis.“ Die Herstellung von Terrakottatafeln mittels Stempeln (mit buddhistischen Motiven und teils Inschriften) war im Gebiet des heutigen Myanmars (bei den Pyu, Mon, Bamar und Arakanesen) lange verbreitet. Die Praxis der Herstellung stammt aus Indien und verbreitete sich mit dem Buddhismus. Die Tafeln seien dabei nicht wegen ihres ästhetischen, sondern wegen ihres religiösen Wertes geschätzt worden - je mehr Bilder mit einem Stempel hergestellt wurden, desto höher war das religiöse Verdienst. Die Stempel bestanden aus einer Kupferlegierung oder aus Terrakotta. Ein Teil der untersuchten bronzenen Stempel stammt aus Myanmar und zeigt Ähnlichkeiten mit Stempeln aus Indonesien, Thailand und Kambodscha auf. Dass in Myanmar diese Stempel längere Zeit in Gebrauch waren als in den Nachbarländern, legt die Vermutung nahe, dass diese Praxis von Myanmar aus an die Länder östlich und südlich weitergegeben wurde.

Jella Fink von der TU Dortmund umreisst in ihrem Promotionsprojekt, das sie in ihrem Referat „Textilien als kulturelles Erbe Myanmars“ vorstellt, drei Themenbereiche: Erstens soll anhand von zwei Fallstudien in Sagaing und in Kyaing Tong die zeitgenössische Textilkultur und die handwerkliche Produktion beleuchtet werden. Zweitens sollen der Wissenstransfer und die Wissensbewahrung von (textil)handwerklichem Können erforscht werden, um die Bedeutung des Konzepts des kulturellen Erbes für Myanmar zu untersuchen. Drittens sollen schliesslich interne und externe Faktoren (Globalisierung, Tourismus, politischer Wandel, ideologischer Wandel, individueller Umgang mit handwerklichem Wissen etc.) beleuchtet werden, die den textilen Produktionsbereich beeinflussen.

Einen Einblick in das politische Handeln der Zivilgesellschaft Myanmars gibt Phuong Le Trong von der Universität Bonn mit seinem Referat „Zivilgesellschaft-Immer noch im Partikularen?“. Die Übertragung des Konzepts von Zivilgesellschaft auf Myanmar ist jedoch äusserst schwierig und unklar. Ein Hauptproblem liegt bereits in der Übersetzung dieses Konzepts, denn dabei gehen Inhalte verloren oder müssen anders erklärt werden. Hinzu kommt, dass die zivilgesellschaftlichen Akteure in Myanmar sehr heterogen sind und mit unterschiedlichen Interessen in dauernder Konkurrenz um knappe Mittel stehen. Gleichwohl lasse sich ein gewisses Potential dieser Akteure für den Demokratisierungsprozess ausmachen. 
Sascha Helbardt von der Universität Passau befasst sich in seinem Referat „Between Old Government Control and New Market Pressure: Myanmar's Media System in the Making“ mit den Medien in Myanmar und ihren Beziehungen zum Staat. Die staatliche Kontrolle und Zensur sind aufgehoben oder zumindest wesentlich gelockert worden. Medien, die aus dem Exil betrieben wurden, kehren nach Myanmar zurück und die Medienwelt wird vielfältiger. Die finanzielle Lage ist dafür angespannt, denn das Geld von Spendern fliesse nun kaum mehr an die (fast) freien Medien (während sie früher als unterdrückte Medien finanziert wurden). Ambivalent ist ausserdem die Beziehung zum Staat, der die Medien einerseits weiterhin zu regulieren, gleichzeitig aber auch internationalen Standards zu entsprechen sucht. So lassen sich zwei Narrative in der Medienwelt in Myanmar finden: Die prostaatlichen und die oppositionellen Medien. Beide Seiten berichteten höchst einseitig, eine Auseinandersetzung zwischen diesen Extremen fehle.

In einer Reihe von Referaten werden anschliessend drei geplante Promotionsforschungen skizziert. Laura Hornig vom Max-Planck-Institut für ethnologische Forschung in Halle befasst sich mit „Religion and moral economy in Myanmar“ und wird dazu in Pathein forschen. Sie versteht die wirtschaftliche Sphäre als ein komplexes Ganzes, das sich nicht auf rationales und profitmaximierendes Handeln von isolierten Individuen und Betrieben reduzieren lässt. Fokus der Forschung soll das soziale, politische und vor allem religiöse Umfeld sein. Sie zielt ein Verständnis der ökonomischen Strategien der verschiedenen Akteure sowie der diesen zugrundeliegenden emischen Auffassungen und Vorstellungen an.

Georg Winterberger von der Universität Zürich befasst sich in seinem Promotionsprojekt ebenfalls mit „Strategien zur Existenzsicherung in Myanmar“. Seine Forschung wird er in Mawlamyine durchführen, da die Stadt sich aufgrund der vielfältigen Möglichkeiten der Einkommensgenerierung und Existenzsicherung für dieses Forschungsprojekt eigne. Sein Fokus liegt auf der Innenperspektive der Akteure, da nur diese das beobachtete Handeln richtig $\mathrm{zu}$ interpretieren erlaube.

Anna Jüschke von der Humboldt-Universität zu Berlin legt ihren Fokus auf die Produktions- und Arbeitsbedingungen in der Textilindustrie in Myanmar. In ihrem Referat „,Made in Myanmar (Burma)‘. Informelle und prekäre Arbeit in der myanmarischen Textilindustrie“ betont sie, dass die westlichen Textilunternehmen, die seit 2014 vermehrt in Myanmar als Produktionsstandort investieren, verstärkt die Produktions- und Arbeitsbedingungen ins Blickfeld rücken. Auch wenn sie die Sozialstandards für den Produktionsstandort Myanmar und den Code of Conduct, dem sich die Textilbranche seit 2015 freiwillig verpflichtet, loben, so deutet die hohe Anzahl von Streiks und Berichten lokaler NGOs aber 
auf problematische Arbeitsbedingungen hin. Jüschke stellt sich insbesondere die Frage, inwiefern die Arbeiterinnen und Arbeiter selbst an den Aushandlungsprozessen beteiligt sind, und welche Handlungsspielräume sie sich zur Vertretung ihrer Positionen aneignen können.

Johanna Neumann von der Humboldt-Universität zu Berlin verbrachte für ihre Promotionsforschung „Looking at Myanmar Society through the Lens of Zatpwe“ sechs Monate mit ,Zat'-Truppen in Myanmar. ,Zatpwe“ ist eines der wichtigsten Genres darstellender Kunst in Myanmar. Die nächtlichen Aufführungen dauern etwa zehn Stunden und werden von anreisenden Truppen auf temporären Bühnen aufgeführt. Die Truppen bestehen durchschnittlich aus 50 Personen. Neumann untersucht in ihrem Projekt, wie ,Zatpwe‘ als Linse benutzt werden kann, durch die die Werte und Normen der Gesellschaft Myanmars sichtbar werden. Gegenstand der Forschung ist daher nicht nur das Bühnengeschehen, sondern auch der gesellschaftlichen Kontext der Veranstaltungen, das, was sich hinter den Kulissen abspielt ebenso wie die Rolle des Publikums.

Esther Tenberg von der Universität Bonn stellt im Referat „Being Tomboy in Yangon - Gesellschaft, Familie und Identität“ den kaum erforschten Themenkomplex rund um die Tomboy - sozial männlich performende, weibliche Personen - vor. Ausgehend davon, dass Gender und Sexualität grundlegende Aspekte zum Verstehen gesellschaftlicher Beziehungen, Normen, Werten und Verhalten sind, stellt sich Tenberg Fragen zu Selbstverständnis, Verhältnis zur Gesellschaft und sprachlichen Mitteln der positiven Identitätsbeschreibung von Tomboys. Erste Ergebnisse zeigen, dass in den Familien der Tomboys zwar durchaus eine mangelnde Akzeptanz herrscht, dass diese aber nicht die von Aktivistinnen und Aktivisten dargestellten Ausmaße annimmt. Auch befänden sich die Tomboys nicht so sehr im Visier der Öffentlichkeit wie beispielsweise die Ladyboys sozial weiblich performende, männliche Personen. Es gebe aber auch kaum in der Gesellschaft akzeptierte Aufgaben oder Berufe für Tomboys - für Ladyboys hingegen schon.

Einen Teil ihrer Forschung über Land- und Eigentumsrechte bei nicht buddhistischen religiösen Gemeinschafen in Yangon stellt Judith Beyer von der Universität Konstanz in ihrem Referat „Kochen auf dem Friedhof. Strategien urbaner Präsenz bei Shiiten in Yangon“ vor. Am Beispiel der Shiiten in Yangon zeigt Beyer auf, wie sich diese Gruppe gegenüber der Öffentlichkeit darstellt, bzw. wie sie von der Öffentlichkeit wahrgenommen wird. Jenseits eines jährlich stattfindenden Rituals ist über die Shiiten in Yangon wenig bekannt. Dies sei zum einen die Folge des strategischen Handelns einzelner Mitglieder und zum anderen die Konsequenz des marginalisierten Status der Muslime in Myanmar. ${ }^{8}$

8 Siehe dazu Beyer (im Druck). 
Worauf der Titel „Nicht-Orte des Erinnerns. Das Gedenken an Aung San in Yangon“ anspielt, erklärt Felix Girke von der Universität Konstanz dem Tagungspublikum: Das Gedenken an General Aung San, der das Land in die Unabhängigkeit führte und im Alter von 32 Jahren ermordet wurde, ist in Myanmar allgegenwärtig. Dennoch findet man Ambivalenzen, wenn man sich die verschiedenen materiellen Repräsentationen von Aung Sans Erbe genauer betrachtet - etwa Geldnoten, Briefmarken, Statuen, Souvenirs, sein Grab, seine Villa, sein Arbeitszimmer. Es ist nach wie vor eine Spannung zwischen Orten und Dingen zu konstatieren: Während Fotos, T-Shirts, Souvenirs etc. lange eine besonders starke Rolle für das Gedenken an den General spielten, waren in der einstigen Hauptstadt Yangon die Stätten seines Wirkens sowie seines Märtyrertods für lange Zeit dem freien Zugang entzogen. Es scheint, dass auch die heutige Regierung noch nicht weiss, wie sie mit diesem Gedenken an den Nationalhelden, deren Tochter die heutige Oppositionsführerin ist, umgehen soll. Felix Girke beobachtete bei den Festlichkeiten anlässlich des „Martyrs’ Day“ am 19. Juli 2015, wie die Öffentlichkeit versuchte, sich diesen Orten wieder anzunähern. In diesem Jahr, in dem Aung San 100 Jahre alt geworden wäre, waren das Museum, die Statue, das Mausoleum, und sogar das „Secretariat“, der Ort der Ermordung, zugänglich, wenn auch teilweise der Zutritt durch viele Verbote erschwert war. Im „Secretariat“ selber blieb das letzte Zugeständnis aus: eine Öffnung des Raums, in dem die Märtyrer starben. Ob diese neue (wenn auch eingeschränkte) Zugänglichkeit zu den Gedenkorten das nicht räumlich gebundene, sondern an bewegliche Objekte geknüpfte Gedenken ablöst, wird sich in den nächsten Jahren weisen. Deutlich wurde jedoch schon dieses Jahr, dass die Öffentlichkeit sich nicht mit der strikten Kontrolle der Gedenkorte durch die Regierung abgefunden hat, und auch, dass das Monopol der politischen Opposition über Aung Sans Bild am Schwinden ist.

Rüdiger Korff von der Universität Passau und Chaw Chaw Sein von der Yangon University geben mit ihrem Referat „Sisyphus or can the university system be reformed?" einen Einblick in die Situation, in der sich die Universitäten Myanmars befinden. Unter der Regierungszeit des Militärs hatte Bildung primär die Aufgabe, den Nationalismus des Landes zu stärken. Heute wollen sich die Universitäten den Herausforderungen, der sich höhere Bildungseinrichtungen durch die Anbindung an eine globalisierte Welt gegenüber sehen, stellen. Dafür sind Reformen im Bildungswesen unumgänglich und integraler Bestandteil des Demokratisierungsprozesses. Diese Reformen sind bereits in Ausarbeitung. Da sie aber noch nicht implementiert sind, und da das alte System bzw. die alten Regelungen heute nicht mehr anwendbar sind, sehen sich die Universitäten momentan in einer schwer zu überbrückenden Kluft, die es so bald als möglich zu schliessen gelte. Die geplanten Reformen stimmten 
jedoch hoffnungsvoll, scheinen sie den Universitäten doch grössere Freiheit zu gewähren.

Am Beispiel des Mythos vom König und der Gerechtigkeitsglocke zeigt Dagmar Hellmann-Rajanayagam von der Universität Passau in ihrem Referat „Wandernde Mythen“ auf, wie Mythen mit dem Buddhismus den Weg von Indien oftmals über Sri Lanka nach Myanmar, Thailand und Kambodscha fanden. Wie kommt es aber, dass ein Mythos - eine oft fiktive Geschichte aus der Vergangenheit, die wichtig für die Gesellschaft ist - von einer fremden Gesellschaft übernommen wird? Beim Mythos vom König und der Gerechtigkeitsglocke handelt es sich um eine sehr alte südindische Erzählung, die aufzeigt, dass ein König das Recht über alles stellen muss und ihm auch sich selbst unterzuordnen hat. Während der Kern des Mythos in seinen Wanderung grundsätzlich erhalten blieb, wurde er in den neuen Gesellschaften in unterschiedliche Erzählstränge geteilt und jeweils verschieden rezipiert, interpretiert und adaptiert. So ist das zentrale Thema dieses Mythos in Myanmar nicht mehr das Recht und die Rechtsprechung, sondern der Herrscher als letzte Rechtsinstanz und die Gerechtigkeit.

Hans-Bernd Zöllner gibt mit seinem Referat „Zwischen Baum und Borke die Rohingya als Spielball antagonistischer Interessen“ einen Überblick über die Rohingya-Frage. Der Anlass für den ersten Teil des Titels, sei es gewesen aufzuzeigen, dass die Geschichte zeige, dass es schon immer unklar gewesen sei, welche Personen oder Gruppen als Rohingya zu bezeichnen seien. Als eine Konsequenz davon ist die Mehrheit der mit dem Begriff bezeichneten Gruppe staatenlos - sie wird von Myanmar als Bürger Bangladeschs angesehen und deshalb als „Bengalis“ bezeichnet, während Bangladesch sie als Bürger Myanmars betrachtet. Sicher ist, dass dieser Begriff in den Listen der britischen Kolonialregierung nicht als Name einer der Ethnien Birmas auftaucht. Es sind gemäss Zöllner drei historische Belastungen auszumachen: 1) Im früheren Königreich Arakan lebten zahlreiche Muslime unter der Herrschaft buddhistischer Könige. Dieses hierarchische System wurde 1784 durch die Integration Arakans in das burmesische Grossreich erschüttert. Nun waren Muslime wie Arakanesen einem fremden König untertan. Eine zweite grundlegende Veränderung ergab sich nach der Integration Arakans in das britisch-indische Kolonialreich. Die früheren Hierarchien wurden nun durch ein Nebeneinander ethnischreligiöser Gruppen ersetzt. Zudem ergab sich nun die Möglichkeit einer verstärkten Einwanderung aus Indien. 2) Der erste Pogrom an Muslimen fand Ende der 1930er Jahren in ganz Myanmar statt, nachdem die muslimischen Inder von Seiten der burmesischen Nationalisten unter Führung von buddhistischen Mönchen als Sündenböcke für die Übel der Kolonialherrschaft ausgemacht worden waren. 3) Während des zweiten Weltkrieges und der Flucht der 
Briten und vieler Inder aus Myanmar fand nach blutigen Auseinandersetzungen zwischen Muslimen und Buddhisten in Arakan eine Trennung der Bevölkerungsgruppen statt. Muslime bildeten die Mehrheit der Bevölkerung im Norden des heutigen Rakhine Staates, während die buddhistischen Einwohner im südlichen Landesteil die Mehrheit bildeten. Diese drei Belastungen führten in Kombination mit einer allgemeinen globalen Angst vor Muslimen und einem durchaus auszumachenden unterschwellig vorhandenen Rassismus in Myanmar zur heutigen Situation. Die etwa eine Million „Rohingyas“ leiden unter einem Grundsatzstreit zwischen den Regierungen Myanmars und des Rakhine States auf der einen Seite und den Interessenvertretern dieser Gruppe auf der anderen Seite um die Frage, ob die Muslime im Norden Rakhines als eine ethnische Gruppe, die schon immer zum heutigen Myanmar gehört habe, anzuerkennen seien oder nicht.

Im abschliessenden Referat „Research Possibilities in Mandalay University“ stellt Aye Thandar Htay von der Mandalay University die Kooperation zwischen der Universität Zürich und ihrer Universität vor. Ausserdem geht sie vertieft auf die Ausrichtung und den Aufbau der Mandalay University ein, sowie auf Kooperationsmöglichkeiten zwischen deutschsprachigen Universitäten und solchen in Myanmar.

Christoph Antweiler von der Universität Bonn schliesslich fasst die Referate und Diskussionen der dreitägigen Tagung zusammen und zeigt Forschungslücken auf: Die Ausgangslage für die Tagung bilde das Interesse an Forschungen in und über Myanmar sowie an Netzwerkbildung. Die Themen waren entsprechend divers und interdisziplinär - vor allem jedoch stammten sie sowohl aus den Sozial- als auch aus den Geisteswissenschaften. Den Schwerpunkt hätten Mikrountersuchungen gebildet. Der Wandel Myanmars sei ebenso aufgegriffen worden wie kulturelle Aspekte. Weniger prominent seien religiöse und politische Themen gewesen. Qualitative Methoden wie Interview und Teilnehmende Beobachtung hätten dominiert, Archivforschungen und Objektforschung seien stark untervertreten gewesen. Als Defizite nennt Antweiler folgende Punkte: Nicht alle Forschenden können die lokale Sprache wirklich sprechen, es sei daher äusserst wichtig, diesen Aspekt in der Lehre zu berücksichtigen. Es gebe bis anhin erst wenig empirische Forschung zu Myanmar, weshalb die zahlreichen Forschungsprojekte ermutigend seien. Ein Problem sei, dass der Mangel an empirischen Forschungen nach sich ziehe, dass für Untersuchungen zum Wandel mangels Daten oft die Basis fehle. Als letzten Punkt bedauert Antweiler, dass zu wenige Forschungen die unzähligen Exilgemeinden und die internationalen Beziehungen Myanmars einbeziehen würden. Myanmar sei nur in seiner Verflechtung mit den Akteuren im Um- und Ausland zu verstehen. 


\section{Bibliographie}

Beyer, Judith (im Druck): „Houses of Islam. Muslims, Property Rights and the State in Myanmar". In: Islam and the State in Myanmar. Hrsg von Melissa Crouch. Oxford: University Press.

Hlaing, U Chit (2008): „Anthropological Communities of Interpretation for Burma. An Overview“. Journal of Southeast Asian Studies 39.2: 239-254.

Myint-U, Thant (2014): „Foreword“. In: 7 Days in Myanmar. A Portrait of Burma by 30 Great Photographers. Hrsg von John Falconer. Singapore: Editions Didier Millet.

Schober, Juliane (2011): Modern Buddhist Conjunctures in Myanmar. Honolulu: University of Hawai'i Press.

Selth, Andrew (2010): „Modern Burma Studies. A Survey of the Field“. Modern Asian Studies 22.2: 401-440.

Steinberg, David I. (2013): Burma/Myanmar. What Everyone Needs to Know. Oxford: Oxford University Press.

Tambiah, Stanley (1975): World Conqueror, World Renouncer. Cambridge: Cambridge University Press.

Universität Passau (2015): Wissenschaftsforum Myanmar (WiMya). http://www.phil.uni-passau. $\mathrm{de} /$ suedostasien/wissenschaftsnetzwerke/wissenschaftsforum-myanmar/ (02/09/2015). 